\title{
Simulation of Heterogeneous Financial Market Model Based on Cellular Automaton
}

\author{
Hong Zhang ${ }^{1}$, Li Zhou ${ }^{1}$, Yifan Yang ${ }^{2}$, Lu Qiu ${ }^{3}$ \\ ${ }^{1}$ School of Information, Beijing Wuzi University, Beijing, China \\ ${ }^{2}$ School of Banking and Finance, University of International Business and Economics, Beijing, China \\ ${ }^{3}$ School of International Business, Nanjing Audit University, Nanjing, China
}

\section{Email address}

dr.yuwenjunxian@gmail.com (Hong Zhang)

\section{To cite this article:}

Hong Zhang, Li Zhou, Yifan Yang, Lu Qiu. Simulation of Heterogeneous Financial Market Model Based on Cellular Automaton. Science Journal of Applied Mathematics and Statistics. Vol. 3, No. 3, 2015, pp. 153-159. doi: 10.11648/j.sjams.20150303.18

\begin{abstract}
In recent years, researchers analyzed the historical data from the financial markets. They found that the statistical result is different from the classical financial theories, models, and methods. The difference is challenging the three hypotheses which are rational people hypothesis, efficient market hypothesis and random walk hypothesis. We need new perspective and tools to re-study the financial market as a complex system. A cellular automata based heterogeneous financial market model is proposed in this categories which dissertation. In this model, the market participant id divided in to two is the fundamentalists and chartists. A learn rules is used to make sure all the market participant can convert in these two categories. The method emulates the interact behaviors between the market participants, and emulates the overall market behavior. The author analyzes the randomness sources, mean-reverting property, bubble happen and bust, and stationary of this model. The author analyzes the relationships between cellular automata based heterogeneous financial market model and the Ornstein-Uhlenbeck model and GARCH models. The data simulated by the financial market model is fit the characteristics such as the fat tail of return's distribution, negative skewness, relationship between return and trading volume, the randomness of volatility, and volatility cluster, which the classical theory is failed to explain. How to add more heterogeneity into the model is discussed in this dissertation. In this dissertation, by using the cellular automata as a tool, an option pricing model and a heterogeneous financial market model are proposed. The result of the option pricing model is close to the result calculated by the formula. The simulation of heterogeneous financial market model can explain many phenomenons which can not be explained by the classical theory, such as the fat-tail of return and the bubble happen and bust. The author also preliminary designs the financial market model based on the asynchronous cellular automata. These models and conclusions indicate that cellular automata have a ability to show the randomness of the financial markets and simulate the behaves of the participants in the financial maket.
\end{abstract}

Keywords: Cellular Automaton, Financial Market, Heterogeneous, Simulation

\section{Introduction}

Y Gao and Czbeni proposed a heterogeneous financial market model with Intrinsic Randomness, which uses the classic Brownian motion model to model the stock price movements. Mantegna and Stanle think Brownian motion model can't explain Thick tail phenomenon and the generation of financial bubbles. You have to relax the hypothesis that the volatility in Brownian motion model is constant if you want to use Brownian motion model to explain Thick tail phenomenon. Based on this idea, Hull and White, Cox. Ingersoll and Ross, Ornstein and Uhlenbeck, respectively, established models of stock price based on stochastic volatility. But the main difficulty for these models is that how to determine the function of the stochastic volatility, in order to make it similar to the changes of market price. Bailia and Bollerslev, Engle, Bollerslev established a volatility model varying with time. Parts of these models explain Thick tail phenomenon.

Y Gao and G beni's model doesn't directly model the price of the stock but model traders' trading strategies and trading volume by the rules of cellular automata. Cellular automata will decide trading strategies according to the present state of cellular to choose their own market behavior. Except the hypothesis that the volatility in Brownian motion model is constant, the price data generated from Y Gao and $\mathrm{G}$ beni's model also has the characteristics of random drift. The 
drift item indicates the expected growth rate of stock price. In classic Black-Scholes model, expected growth rate is replaced by the risk-free interest rate under the risk-neutral conditions. This paper argues that, because the differences in traders' information level, risk-bearing capacity, budget constraints and trading strategies will lead to the differences among traders' expectations on growth rate of securities, it would be better to replace the fixed drift rate by the random drift rate. The randomness of the drift rate produced by the overall behavior of heterogeneous traders makes cellular automata to better simulate the price changes in this market.

The efficient market hypothesis assumes that market's participants are rational in the process of making decisions, however, that is not the case in the real market. In 1987, for example, the collapse of the financial market and the Internet bubble's burst. This theory- inconsistent irrationality makes researchers began to notice the behavioral factors in the stock price formation mechanism. Many researchers began to believe that irrational psychology, such as greed, fear, and so on will have a huge impact on the behavior of market's participants. In the efficient market hypothesis, some researchers added functions of rational utility to reflect the effect of market's participants ' behavior on stock prices. This paper argues that heterogeneity is the main internal cause of the change of the price. The most famous financial model studying heterogeneity divided traders into three types, fundamentalists, chartist and noise trader. This paper follows this classification but attributes the noise traders to Brown motion's random item, which means only consider fundamentalists and chartists.

In the heterogeneous model set up by Follmer Horst and Kirman, some financial experts, who make trading strategies by fundamental asset or technical analysis, provide traders with trading strategies. Traders choose investment strategy according to financial experts' performance, which can be calculated from the experts' historical data of their investment income. The most important conclusion of this model is that the mutual transformation between fundamentalists and chartist explains the cause of the financial bubble's burst. If the external noise items enter the market, the value level of experts on fundamental asset value orientation will be lower and that on technical analysis orientation will be higher. Then bubble is produced. This kind of change of investment strategy in market leads to pursuit-risen-up phenomenon (Phenomena of Chasing Rising Stocks) in market and shares soared on the stock exchange (Bubble formation). In order to avoid this soaring price, model sets up upper bound in the number of participants choosing pursuit-risen-up strategy. So in the long run, investment strategy on fundamental asset value orientation will occupy the market again and the bubble will burst. Then stock prices will be stabilized. This heterogeneous model proved the existence and uniqueness of the financial market price equilibrium .However, there are two things the model doesn't take into account. First, the model does not consider the price changes as a result of internal market mechanism, so bubble's formation and collapse are both caused by external factors. The formation of the bubble needs an external noise into the market and reduce the performance of experts on fundamental asset value orientation. The collapse of the bubble is in the need for an external upper limit imposed on technical analysis oriented trading strategy. Cellular automaton model does not need these external factors to link trading strategies with current stock status. The evolution of the trading strategy can produce within financial bubble's generation and burst., in no need of setting any upper bounds. Second, the model shows that when chartists are the majority in the market, stock prices will show huge volatility. But it does not indicate the mathematical relationship between the number of chartists and stock fluctuations. The model does not give any analysis result to describe how the stock fluctuations cause bubbles and make them burst. But Cellular automata model, can give analysis results of bubbles' formation and burst in functional form of trading strategy by simulation.

\section{Simulation of Heterogeneous Financial Market Model Based on Cellular Automaton}

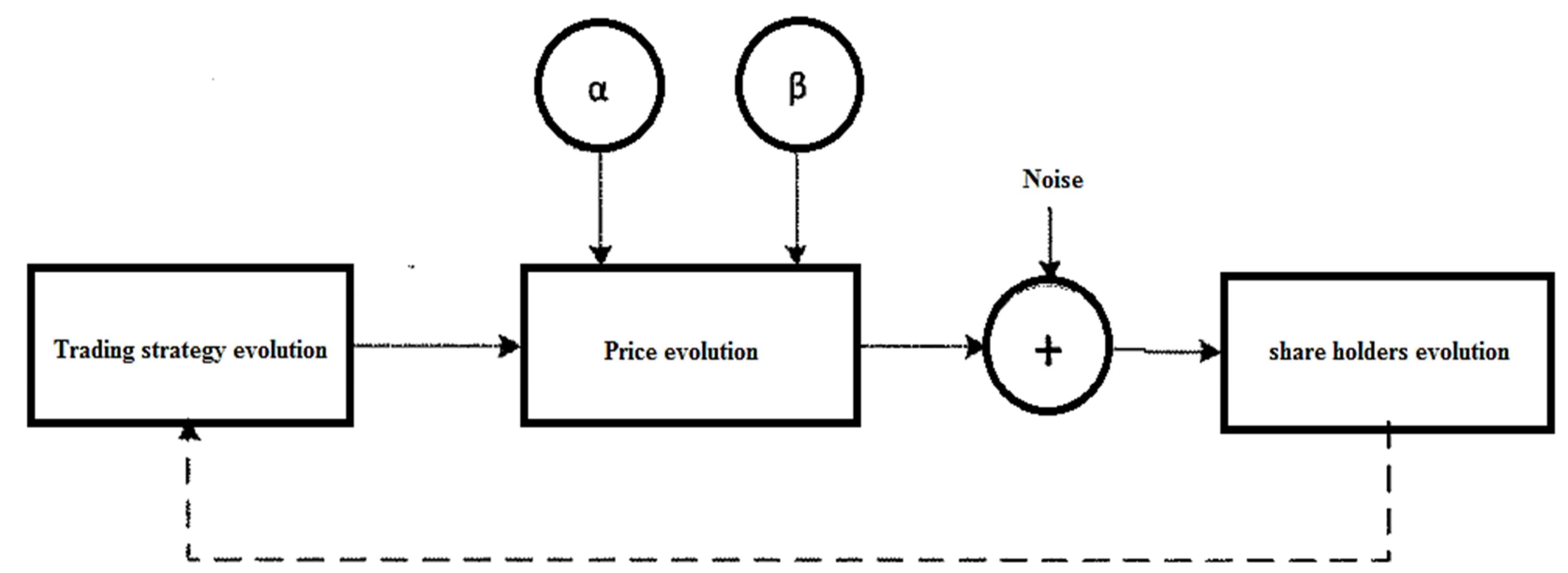

Fig. 1. System module of heterogeneous financial markets model based on the cellular automata. 
This section describes the use of heterogeneous financial market model based on cellular automata to implement model evolution and record the changes in prices. Figure 1 illustrates the modules of the simulation system. The direction of the arrow represents the order of variables. The dotted arrow indicates the self-learning process of cellular automaton model

To illustrate this process, the following describes the evolution from the time $t$ to time $t+1$. At the time $t$, the system variables include $\mathrm{H}(\mathrm{x}, \mathrm{t})$ and $\log \mathrm{P}(\mathrm{T})$; after cellular automaton evolution, culminating in the $\mathrm{t}+1$ time for the evolution of new variables, $\mathrm{H}(\mathrm{x}, \mathrm{t}+\mathrm{l})$ and $\log \mathrm{P}(\mathrm{T}+1)$. The steps are as follows:

1. First of all, according to the formula (1), based on $H$ (x, t) updating $L(x, t)$.

$$
\begin{gathered}
P\{L(x, t)=F\}=\max (0, \min (1, H(x, t))) ; \\
P\{L(x, t)=C\}=1-P\{L(x, t)=F\} ;
\end{gathered}
$$

2. According to the formula (2) to calculate the value of $A$ (T), B (T) and C (t).

$$
\log p(t+1)=\frac{A(t)}{1-B(t)} \log F+\left(1-\frac{A(t)}{1-B(t)}\right) \log p(t)+\frac{\sigma(t)}{1-B(t)} d W_{t}
$$

where

$$
\begin{aligned}
A(t) & =\frac{\int H(x, t) L(x, t) d x}{\int H(x, t) d x} \alpha \\
B(t) & =\frac{\int H(x, t)(1-L(x, t)) d x}{\int H(x, t) d x} \beta \\
\sigma(t) & =\frac{\int H(x, t) \sigma(x) d x}{\int H(x, t) d x}
\end{aligned}
$$

3. According to the formula (2) to calculate the market price of $\log \mathrm{P}(\mathrm{T}+1)$

4. According to the formula (3) to calculate the traders reference value $\log \mathrm{R}(\mathrm{x}, \mathrm{t})$

$$
\begin{aligned}
& \log R(x, t)=\log p(t)+\alpha L(x, t)(\log F-\log p(t)) \\
& +(1-L(x)) \beta(\log p(t+1)-\log p(t))+\sigma(x) d W_{t}
\end{aligned}
$$

5. According to the formula (4) to calculate the shareholding $\mathrm{H}(\mathrm{x}, \mathrm{t}+\mathrm{l})$ at the $\mathrm{t}+1$ moment .

$$
H(x, t+1)-H(x, t)=H(x, t) \log \left(R(x, t) / p_{M}(t)\right)
$$

To be able to simulate, the initial parameters of the model are given as the following:

1. The number of cellular automata model.

2. The number and length of time steps the evolution.

Fixed parameters: the value of the underlying assets, $\log$ F.

Fluctuation scale factor of each cellular.

Initial value of share holders $\mathrm{H}(\mathrm{x}, \mathrm{t}=0)$, the initial price $\log \mathrm{p}(\mathrm{t}=0)$

The following simulation is based on a standard normal distribution, which has the qualifications: cellular number of 50 , the initial price of $\log \mathrm{P}(0)=2$, fixed parameters $\mathrm{a}=0.5$ and $b=0.5$, the value of the underlying assets of $\log F=3.0$. The noise term for each step of the $\mathrm{D}$ is not from the mean of 0 to 1 of the variance of the standard normal distribution.
The fluctuation ratio factor $\mathrm{C}(\mathrm{x})$ is from the range of $[0,1]$, a uniform distribution, and remain unchanged in the evolution process of the whole. The initial value of the shareholding of $\mathrm{H}(\mathrm{x}, \mathrm{t}=0)$ is extremely important for the whole evolutionary process. As discussed in the previous section, the total investment capital of $\mathrm{C}$ is not only an important parameter in the process of price changes. but also determines which has the dominant position in the market potentially, basic asset-oriented or technology analysis-oriented. Setting the initial share rightly can get different patterns of market evolution: the underlying asset-oriented market, technology analysis -oriented market and conversion market

For each type of market structure, this paper gives three kinds of image: price trajectory image, technical analysis and price volatility factor comparison image and the image of drift rate and volatility. In these images, we have the solid line representing the random fluctuations of Wiener process, dotted line representing the random drift rate of every step and the dotted line representing the price change. The underlying asset-oriented market.

\section{The Analysis of Underlying Asset Value-Oriented Dominant Market}

Suppose that at the initial state, shares holding $\mathrm{H}(\mathrm{x}, \mathrm{t}=0)$ takes a uniform value in the interval $[0,1]$. Cellular automata model is in the underlying asset value-oriented dominant market. The features of this market are as following:

1. Prices return to the underlying asset value.

2. Stochastic volatility of Wiener process is small because of the low factor of technical analysis.

3. The random drift rate and random Wiener process offsetting each other makes smaller fluctuation in price. As shown in figure 3, random drift rate and random Wiener process present offsetting trends which results in a relatively stable price trajectory. 

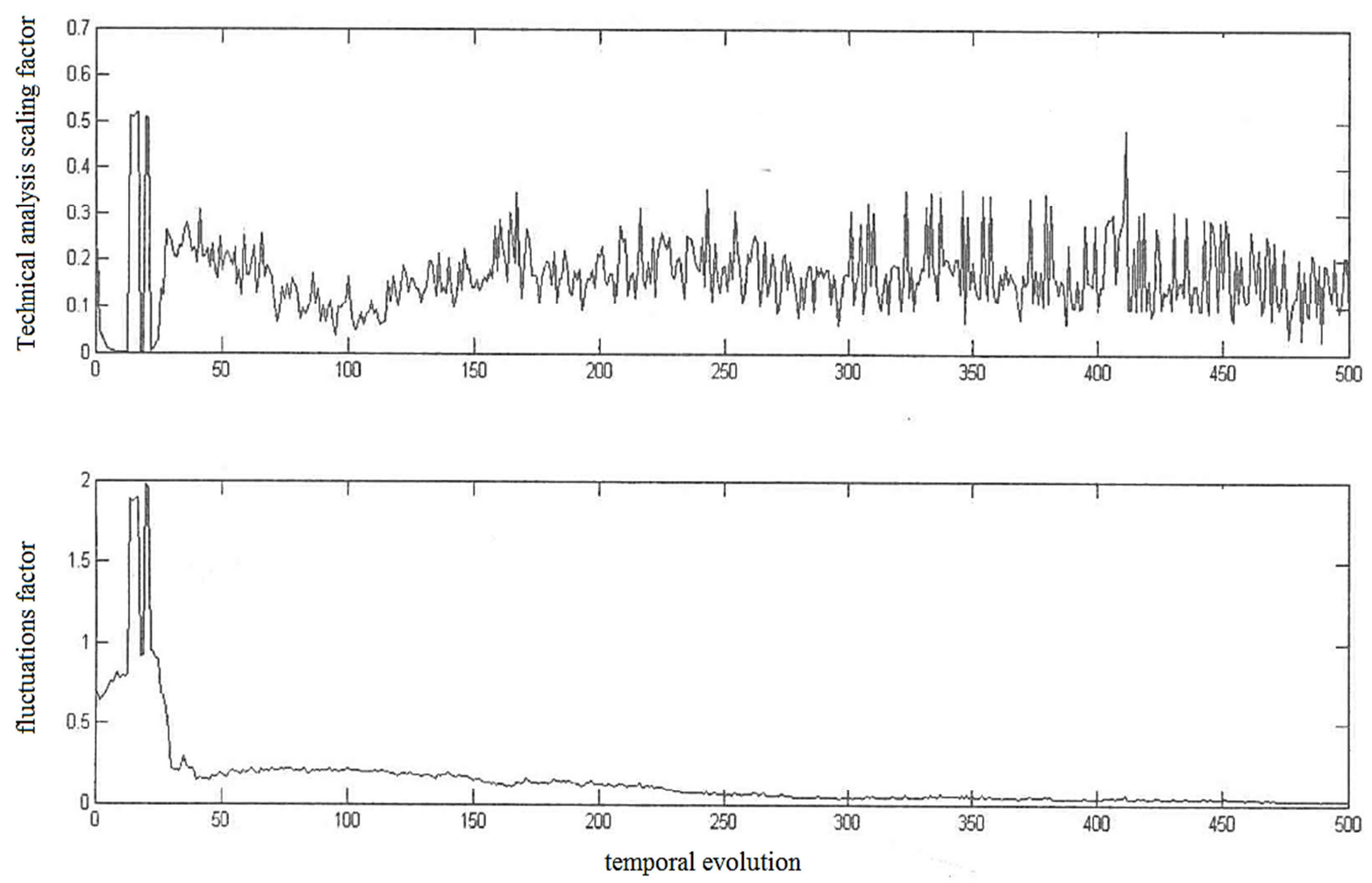

Fig. 2. Charted factor and fluctuations factor in the fundamentalist-dominated market.

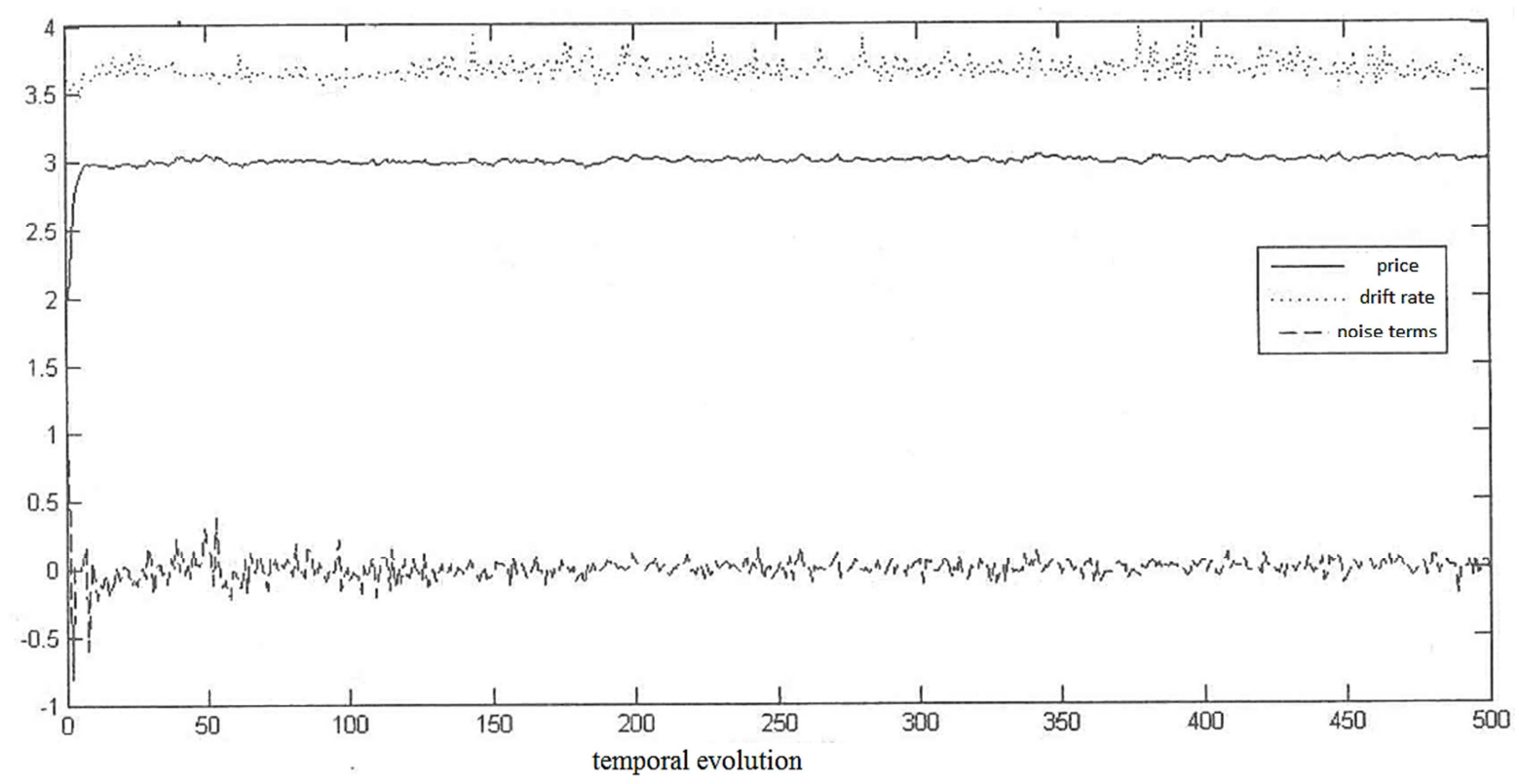

Figs. 3. Drift rate, noise terms and price trajectory in the fundamentalist-dominated market.

\section{The Analysis of Technology Analysis -Oriented Market}

Suppose that at the initial state, shares holding $\mathrm{H}(\mathrm{x}, \mathrm{t}=0)$ takes a uniform value in the interval. The market is occupied by technical analysis investors. The features of this market are as following:
1. It shows greater volatility in the price curve and sometimes produces foam.

2. Stochastic volatility of Wiener process is greatly high due to the high factor of technical analysis,

3. There was no offset relationship between the random drift rate and random Wiener process. Therefore, the price has a great volatility. 

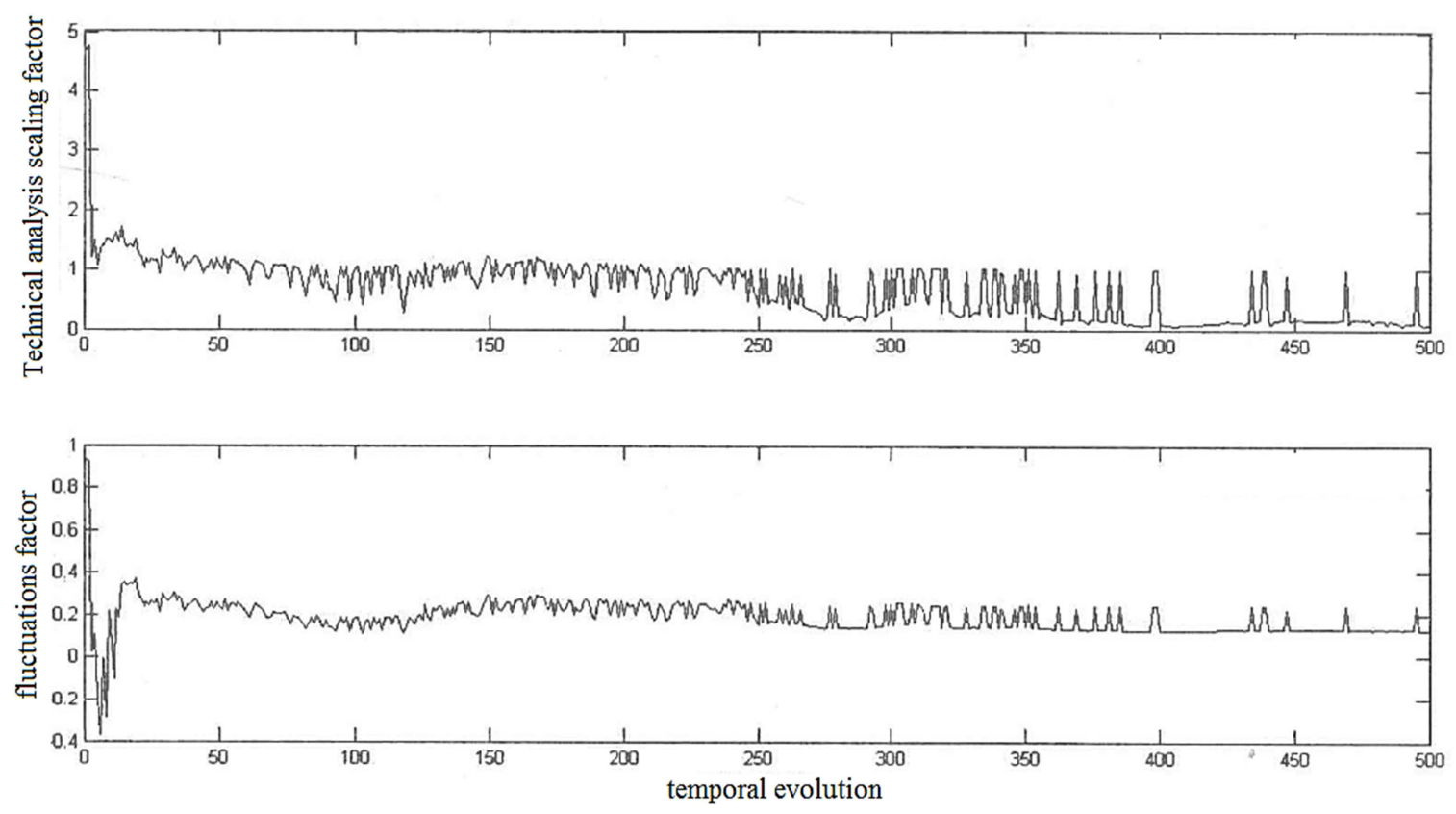

Fig. 4. Charted factor and fluctuations factor in the chartists-dominated market.

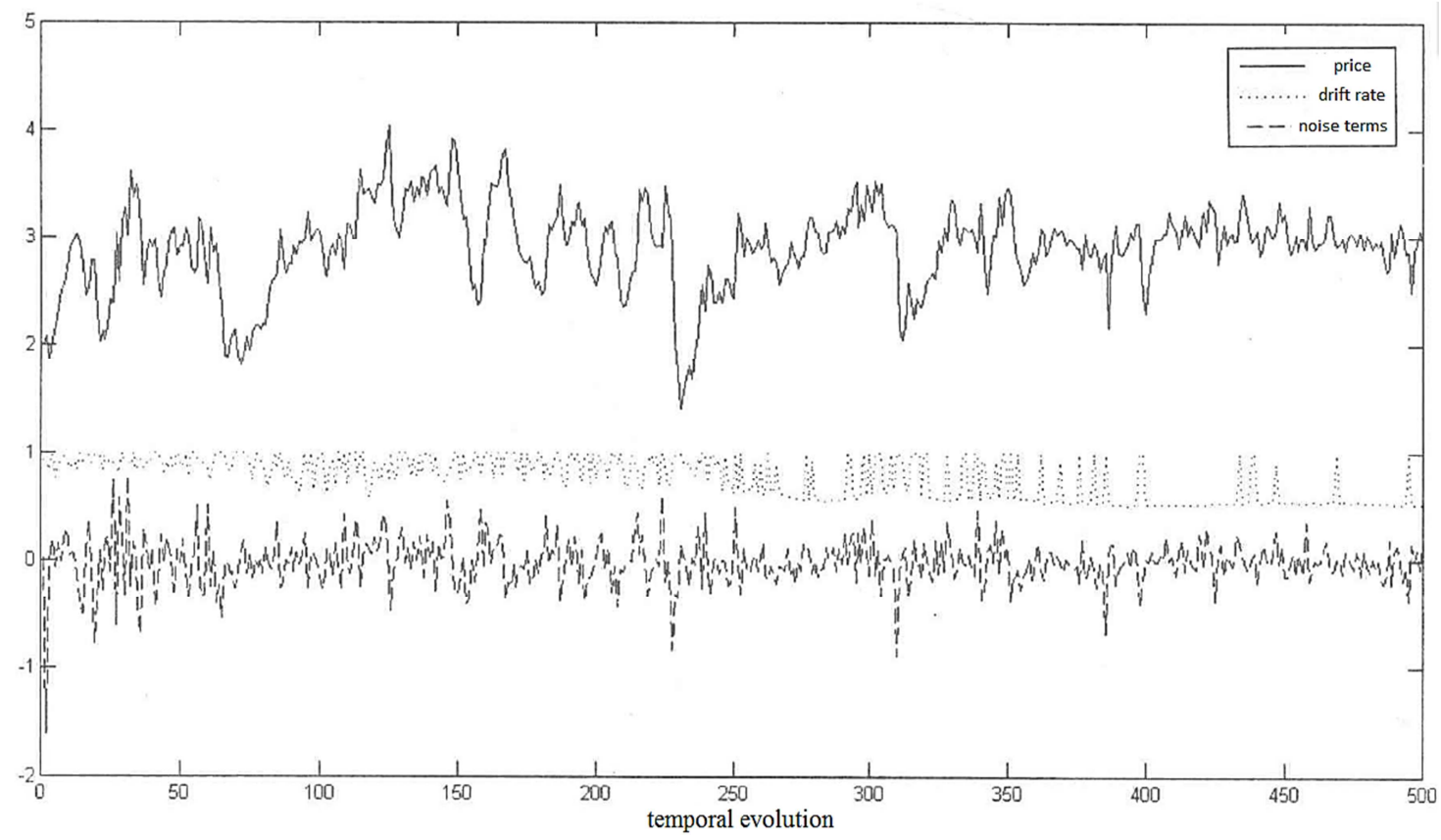

Fig. 5. Drift rate, noise teens and price trajectory in the chartists-dominated market.

\section{The Analysis of Conversion Market}

Suppose that at the initial state, shares holding $\mathrm{H}(\mathrm{x}, \mathrm{t}=0)$ takes a uniform value in the interval $[0,0.5]$. Then in the market, the underlying assets value-oriented and technology analysis-oriented alternately dominated this market. The features of this market are as following:

1. Prices show the relative volatility and jumps situation, but in the long run, the price has tendency to return to the basic value.

2. Fluctuations are larger when the underlying assets value-oriented dominated the market than the technology analysis-oriented.

From the comparison of the stochastic volatility line of the Wiener process and the drift rate of conversion market, the market shows a characteristic of the technical analysis and the mutual effect between the random drift rate and random Wiener process is not obvious from the first 100 to 200 time steps. The market shows the underlying asset value-oriented features when more than this time zone. Random drift rate and random Wiener process begin cancel each other out and prices begin return to value. 

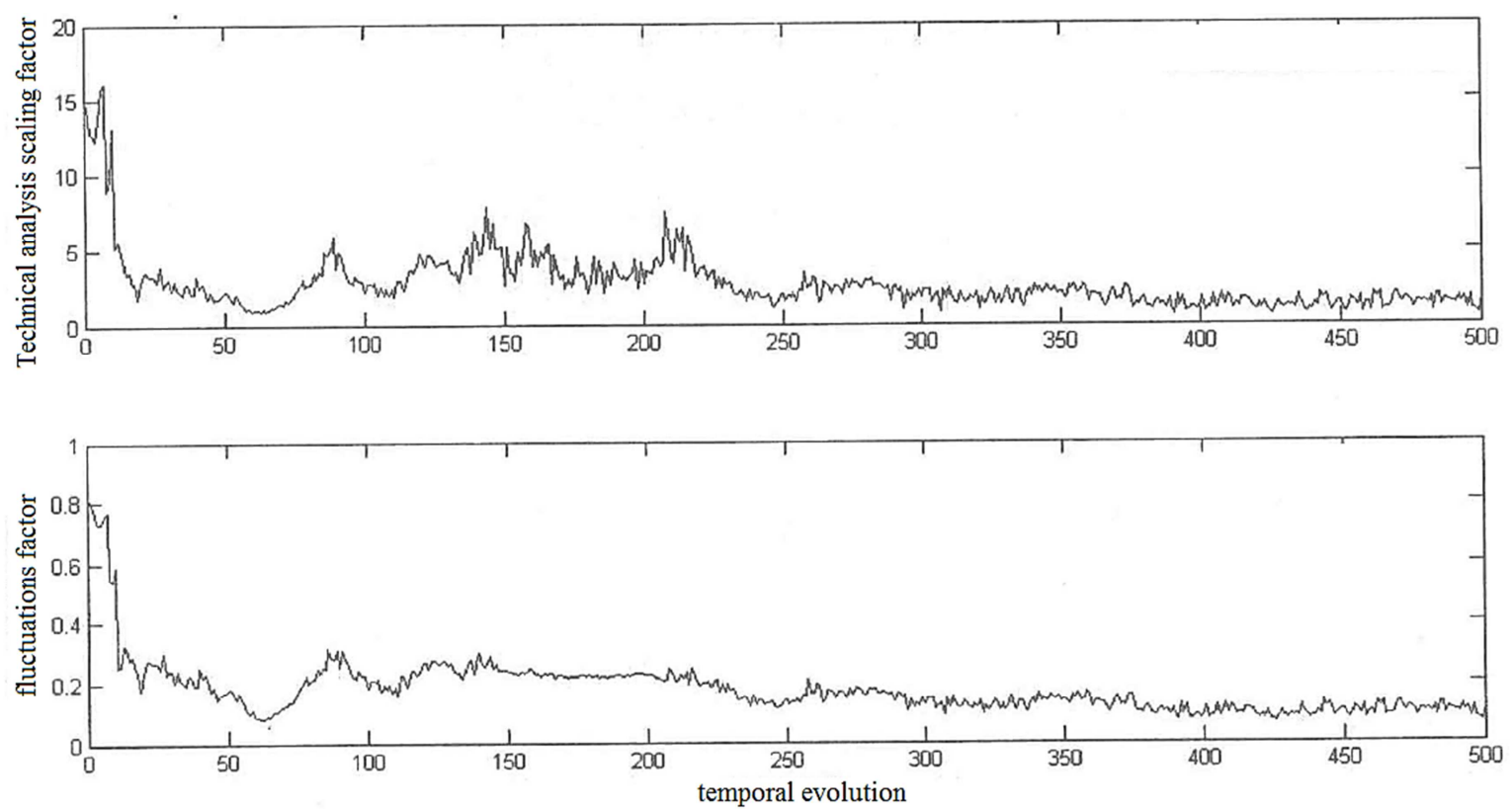

Fig. 6. Charted factor and fluctuations factor in the transform market.

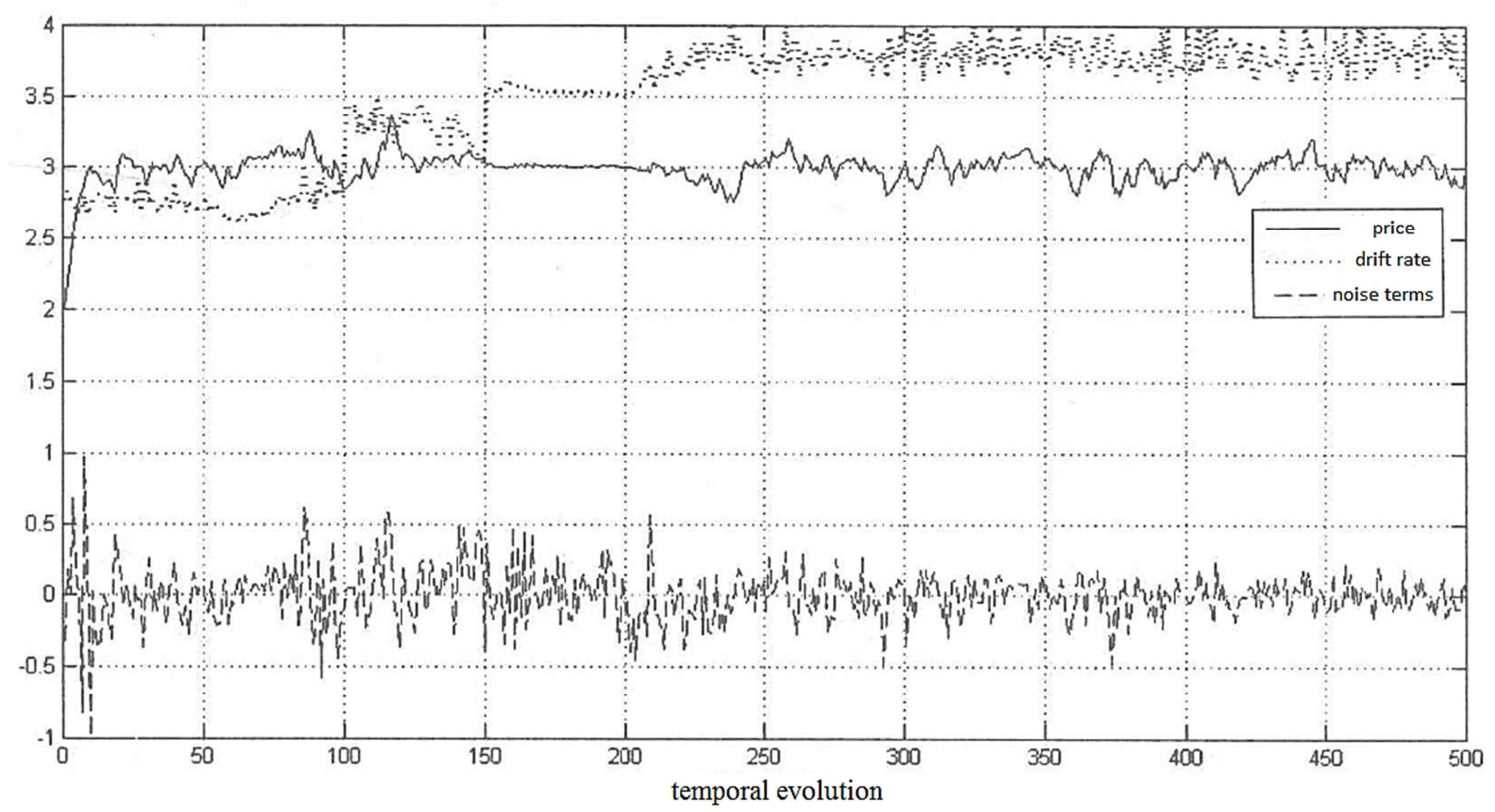

Figs. 7. Drift rate, noise terms and price trajectory in the transform market.

\section{Summary}

The features of the underlying asset value-oriented dominant market are as following:

1. Prices return to the underlying asset value.

2. Stochastic volatility of Wiener process is small because of the low factor of technical analysis.

3. The random drift rate and random Wiener process offsetting each other makes smaller fluctuation in price. As shown in figure 3 , random drift rate and random Wiener process present offsetting trends which results in a relatively stable price trajectory.

The features of the technology analysis -oriented market are as following:
1. It shows greater volatility in the price curve and sometimes produces foam.

2. Stochastic volatility of Wiener process is greatly high due to the high factor of technical analysis,

3. There was no offset relationship between the random drift rate and random Wiener process. Therefore, the price has a great volatility.

The features of the conversion market are as following:

1. Prices show the relative volatility and jumps situation, but in the long run, the price has tendency to return to the basic value.

2. Fluctuations are larger when the underlying assets value-oriented dominated the market than the technology analysis-oriented. 


\section{Acknowledgements}

This paper is funded by the project of National Natural Science Fund, Logistics distribution of artificial order picking random process model analysis and research(Project number: 71371033); and funded by intelligent logistics system Beijing Key Laboratory (No.BZ0211); and funded by scientific-research bases--Science \& Technology Innovation Platform---Modern logistics information and control technology research (Project number: PXM2015_014214_000001); and funded by 2014-2015 school year, Beijing Wuzi University, College students' scientific research and entrepreneurial action plan project (No.68); and funded by Beijing Wuzi University, Yunhe scholars program(00610303/007); and funded by Beijing Wuzi University, Management science and engineering Professional group of construction projects. (No. PXM2015_014214_000039). University Cultivation Fund Project of 2014-Research on Congestion Model and algorithm of picking system in distribution center (0541502703)

\section{References}

[1] Gao Y Beni G Price Fluctuations in Market Model with Heterogeneous Trading Strategies. Proceedings of tha 4th IASTED: Financial Engineering and Application, Berkeley CA, 2007.

[2] Gao Y, Beni G. Model of Heterogeneous Market wiih Intrinsic Randomness. International Conference on Computational Intelligence in Economics \& Finance, Salt Lake City, UT, 2007.

[3] Barndorff-Nielsen O E, Shephard N. Non-Gaussian Ornstein Uhlenbeck-based Models and Some of Their Uses in Financial Economics [J]. Journal of the Royal Statistical Society, Series B, Vo1. 63(2), 2001, 167-241.
[4] Engle R E. Autoregressive Conditional Heteroskedasticity with Estimates of the Variance of United Kingdom Inflation [3]. Econometrics, Vo1.50, 1982, 987-1007.

[5] Bollerslev T. Generalized Autoregressive Conditional Heteroskedasticity [J]. Journal of Econometrics, Vol. 3(1), 1986, 307-328.

[6] Malkiel B G. The Efficient Market Hypothesis and Its Critics [J]. The Journal of Economic. Perspectives, Vo1. 17(1), 2003, 59-82.

[7] Lux T. The Socio-economic Dynamics of Speculative Markets: Interacting Agents, Chaos, and the Fat Tails of Return Distributions [J]. Journal of Economic Behavior and Organization, Vo1.33 (2), 1998, 143-165.

[8] Chairella C, Dieci R, Gardini L. Asset Price Dynamic in a Financial Market with Fundamentalist and Chartists [J]. Discrete Dynamics in Nature and Society, Vol. 6(2), 2001, 69-99.

[9] Horst U,. Financial Price Fluctuations in a Stock Market Model with Many Interacting. Agents [J]. Economic Theory, Vo1. 25(4), 2005, 917-932.

[10] Follmer H, Horst U, Kirman A. Equilibria in Financial Markets with Heterogeneous. Agents: A Probabilistic Perspective [J], Journal of Mathematical Economics, Vo1. 41(1-2), 2005, 123-155.

[11] Uhlenbeck G E, Ornstein L S. On the Theory of Brownian Motion [J]. Physical Review. Letters, Vo1.36, 1930, 823-841.

[12] Feller W. An Introduction to Probability Theory and its Applications [M], Wiley, 1958.

[13] Larralde H. Statstical Properties of a Discrete Version of the Ornstein- Uhlenbeck, Process [J]. Physical Review E, Vo1.69, 2004, 027102.

[14] Vasicek O A. An Equilibrium Characterization of Tenn Structure [J]. Journal of Financial, Economics, Vo1.5, 1977, 177-188. 